\title{
Fog Computing in the context of Smart Home, voice assistant and the future of IoT
}

\author{
Fog Computing en el contexto de Smart Home, asistente de voz \\ y futuro de IoT
}

\author{
Javier Pinzón Castellanos ${ }^{1}$ (iD) and Miguel Antonio Cadena Carter ${ }^{1}$ (DD \\ ${ }^{1}$ Engineering Faculty, Universidad Autonoma de Bucaramanga, Bucaramanga, Colombia. \\ jpinzon408@unab.edu.co,mcadena@unab.edu.co
}

(Received: April 10, 2019; accepted: December 13, 2019)

\begin{abstract}
Fog Computing is the distributed computing layer that lies between the user and the cloud. A successful fog architecture reduces delay or latency and increases efficiency. This paper describes the development and implementation of a distributed computing architecture applied to an automation environment that uses Fog Computing as an intermediary with the cloud computing layer. This study used a Raspberry Pi V3 board connected to end control elements such as servomotors and relays, indicators and thermal sensors. All is controlled by an automation framework that receives orders from Siri and executes them through predetermined instructions. The cloud connection benefits from a reduced amount of data transmission, because it only receives relevant information for analysis.
\end{abstract}

Keywords: Fog Computing, Cloud Computing, Internet of Things, Distributed Computing, Smart home.

Resumen. Fog Computing es la capa de computación distribuida más próxima al usuario. Una arquitectura fog exitosa permite reducir el retardo o latencia y aumenta la eficiencia. En este artículo se expone el desarrollo e implementación de una arquitectura de computación distribuida, aplicada a un entorno de automatización que aprovecha el Fog Computing como intermediario con la capa de computación en la nube. El estudio utilizó una placa de desarrollo Raspberry Pi V3 conectada a elementos finales de control como servomotor y relés, indicadores y sensores térmicos. Todo controlado por un framework de automatización que recibe las órdenes de Siri, las cuales son ejecutadas por medio de instrucciones previamente determinadas. La conexión a la nube se beneficia al reducirse el envío de datos ya que ahora solo se recibe la información relevante para su análisis.

Palabras clave: Fog Computing, Cloud Computing, Internet de las Cosas, Computación distribuída, Casa inteligente.

\section{Introduction}

Fog Computing has sparked substantial academic interest because it enables separating processes performed in the cloud, thereby enabling savings in investment in information and communications technologies. Cloud Computing storage and processing have grown, which has led to price increases. In response, an architectural design has been developed in order to perform local processing and thereby reduce cloud consumption. Based on the above, a study and concept test were carried out using the Fog Computing architecture, consisting in an implementation with hardware and free software, identifying the various modules used for communicating and linking them to each other. Consequently, a Fog architecture implementation process was proposed for academic purposes, in order to understand the performance,

Cite this work as Pinzón Castellanos J., Cadena Carter M. (2020). Fog Computing in the context of Smart Home, voice assistant and the future of IoT. Revista Colombiana de Computación, 21(1), 6-12. https://doi.org/10.29375/25392115.3894 
operation and separation between new computing trends such as the Internet of Things (IoT) and Dew Computing, which are included within a new distributed computing architecture.

Based on a literature review, the project is based on the themes set forth in each article discussing deficiencies on topics that can be addressed through research. The concept of Fog Computing is defined by NIST in its special issue SP-500-325, which highlights that fog computing is a model based on layers that enables ubiquitous, continues, shared and scalable access for IT resources that facilitates the deployment of distributed elements with reduced latency in applications and services (Iorga et al., 2018). It coincides with the distributed computing architecture and references a case of a smart gateway that contributes to the theoretical framework of layers to determine each one. Additionally, several of the reviewed authors conceive Fog Computing as an extension of Cloud Computing, where the sensitivity of the information, the qualities and the comparison between cloud computing and the fog architecture and the Internet of Things are critical points highlighted as topics to be addressed by this project (Bakhshi, Rodriguez-Navas, \& Hansson, 2019).

A proposed domotics solution that uses a Raspberry Pi and the Apple voice recognition module called Siri, is a support point to develop the Fog Computing architecture, which enables managing the architecture (Wei \& Wu, 2019). A study of the companies that develop Fog Computing implementations is a contribution for its approval in the industrial sector, where research is performed, and concepts are set forth on the computing layer and a conceptual application is developed in the area. Additionally, success cases and implementations are reviewed, particularly in the area of medicine (Isa, Musa, El-Gorashi, \& Elmirghani, 2019).

A highlighted concept is mobile Fog Computing, and the authors present the qualities and compare them with the IoT, proposing a development model (Wang et al., 2019). The contribution to the research process provides several points of view, including scenarios of related architectures such as Cloud Computing, Dew Computing and the Internet of Things (Skala, Davidovic, Afgan, Sovic, \& Sojat, 2015), thereby projecting various themes between computing architectures and IT developments.

Edge computing is presented with qualities and structures related to security in a study performed with the man-in-the-middle technique, which highlights the security criteria that should be taken into consideration to build a Fog Computing architecture (Tsigkanos, Avasalcai, \& Dustdar, 2019). Lastly, a concept and limited IoT challenge is presented, including as applicable: the modernization of web services, their development and adaptation (Zhang, Jin, Zheng, \& Yang, 2019).

\section{Description of the System}

Figure 1 displays an overview of the system, which consists of the proposed Fog Computing platform and the various associated devices, including the type of connection and interface used. The requests are processed using the described arrangement in the Fog platform, which displays each component involved in the architecture, in order to ensure the clarity of the requests made.

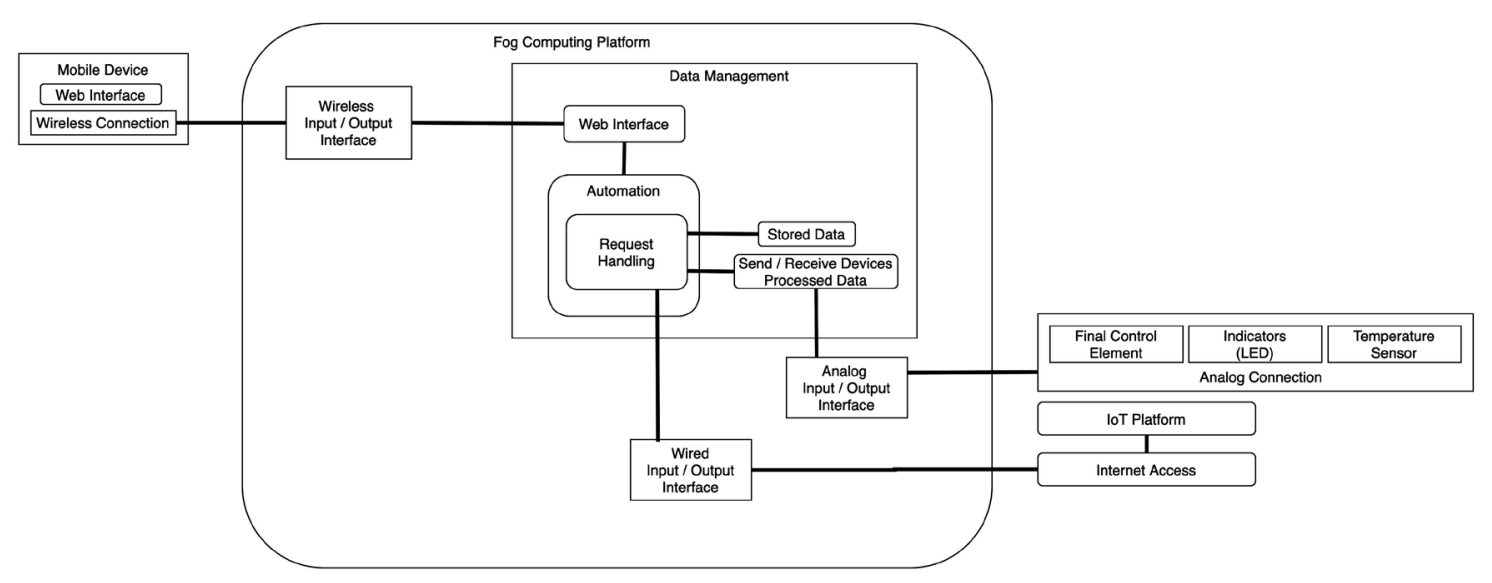

Figure 1. Overall diagram of the Fog Computing system 
The system's information management component consists of organizing the data to enable their processing, storage, viewing and exporting to another system. It includes queries of the stored data and includes a report generation option.

The automation block consists in automating certain actions, in order words, for the actions to be performed automatically without user intervention. This is the reason why this concept of an automated system is often used in industrial environments, where the data are processed and turned into valuable information for the user.

The most noteworthy component is the requests manager, which performs automatic tasks such as acquiring, storing, viewing and exporting data once they have been assigned, as long as the origin of the request is configured.

\section{Hardware Design}

The proposed system design uses devices that include a low-cost processing component. It was therefore decided to use single-board resources developed with special features that are similar to a functional computer, i.e., they contain a processor, RAM memory, graphics card, connectivity, and analog inputs and outputs. The latter are to enable the use of sensors and other components that interact with the single board. It is desirable to include wireless connection in the system, as it resolves the connectivity issue while reducing space and avoiding the use of additional components.

The following boards were considered as options: Raspberry Pi 3 model B and Banana Pi M2+. The selection criteria assigned greater importance to processing capacity, due to the automation processes. Consequently, the Raspberry Pi 3 model B was selected, due to its greater speed performance. The Banana Pi M2+ has 32 bits whereas the Raspberry Pi 3 model B has 64 bits, which enables managing greater amounts of information in the architecture. Additionally, the Raspberry Pi 3 model B was selected because it is a single board with greater maturity in the academic community, its free hardware and software, and its higher impact in the development architecture (IoT Eclipse, 2019).

Three types of elements were used to build the architecture: final control elements such as servo motor, LED indicators and temperature sensor, in line with the industry's most common components (see Figure 2).

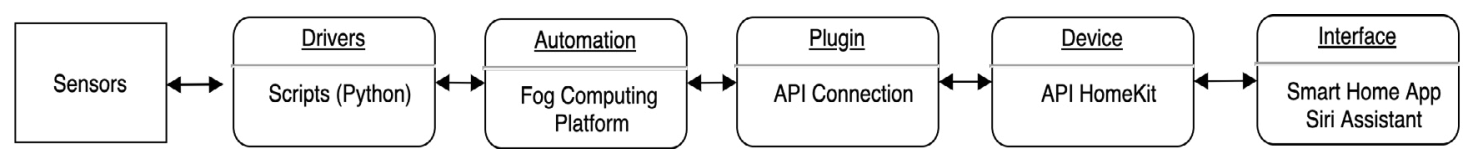

Figure 2. Connections between work tools

Each component is displayed, indicating its features. The selected final control element was the Servo Motor HK15138 by HobbyKing, which meets the required specifications. A $5 \mathrm{~mm}$ LED indicator was selected. The selected DHT11 temperature sensor is fully compatible with the Raspberry Pi 3 model B, because it includes the controllers. The sensor's pins are distributed as +VCC (3.3V), GND (Ground) and S (Signal).

\section{Software Design}

The selection of the software tools was based on their convenience for the Fog Computing architecture, including the operating system and the complementary software.

\subsection{Operating System}

The Raspbian operating system based on Debian was used because it has the libraries, controllers and modules that the Raspberry Pi requires to correctly operate within the proposed platform. 


\subsection{Complementary Software}

The programming language was selected based on the sensors and their controllers, in order to achieve problem-free linkage and clear code reading.

Automation programs were selected that use the Python programming language as desired option. This is in order to meet the aforementioned requirements and because it is the most used language for industrial automation and IoT, as demonstrated by the review performed by bodies in charge of developing IoT (IoT Eclipse, 2019).

The connections diagram in Figure 2 displays each framework, namely the sensors with controllers, connected and managed by the automation model, to which a complement is attached that provides a bridge towards the iOS device that connects with the API HomeKit (Homekit, 2016). After this, Siri, the voicecontrolled personal assistant interacts with the components set up in the Home application, and lastly the requests are sent to the various work blocks.

\section{Diagram of requests between Fog Computing and the Internet of Things}

A diagram was prepared to define the environment for the proposed Fog Computing architecture, showing the different types of requests and areas involved. In order to monitor the petitions, the proposed platform was compared to an IoT platform.

\subsection{Fog Computing}

Figure 3 displays an arrangement of requests aimed at guiding the researcher on how to make the various requests from various roles, where the main player is the user and the proposed Fog platform is the main receiver. For this reason, each ancillary role is defined as an element of automation and the IoT platform, because they are both part of the Fog Computing architecture.

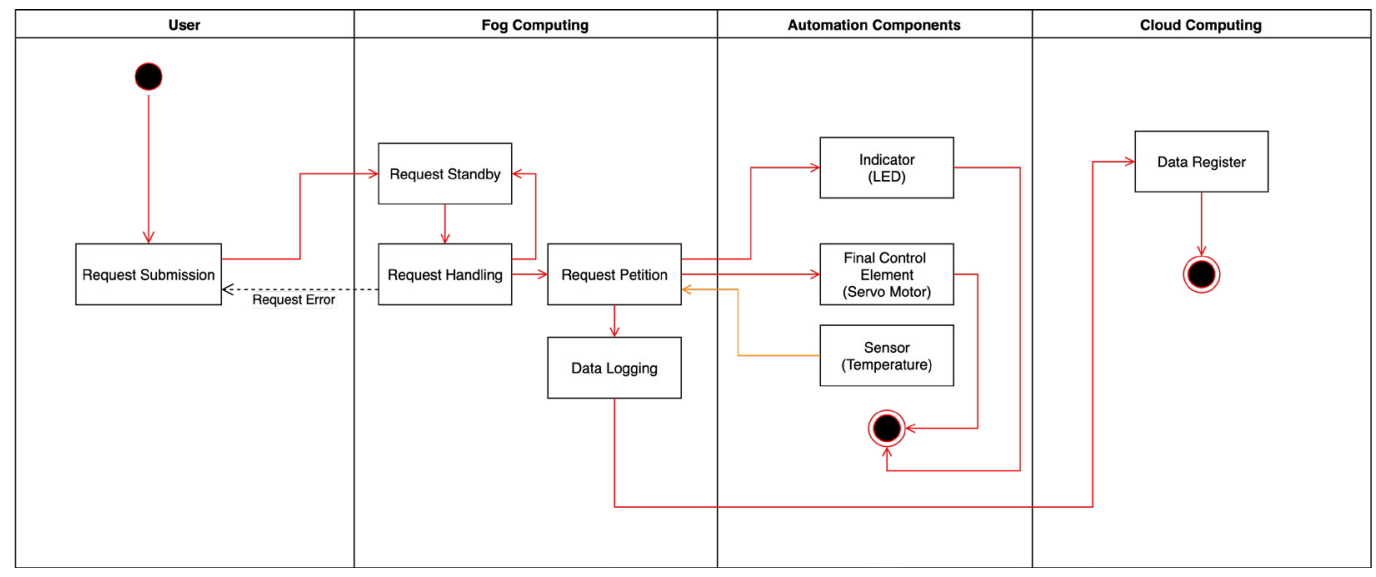

Figure 3. Diagram of Fog Computing requests

Different components are selected as elements for automation, represented in different data inputs in the execution module, which implies delivery of the data only when it is requested, and for this reason it is named differently. The cloud receives the data from the Fog Computing architecture, because it receives data that has already been processed for recording or storage.

\subsection{Internet of Things}

Figure 4 displays an arrangement of requests for IoT, which tells the researcher how the different requests are made by the various IoT roles, where the main player is the user, who sends the various requests to the cloud computing layer where the IoT architecture is hosted, becoming the receptor of the requests. 


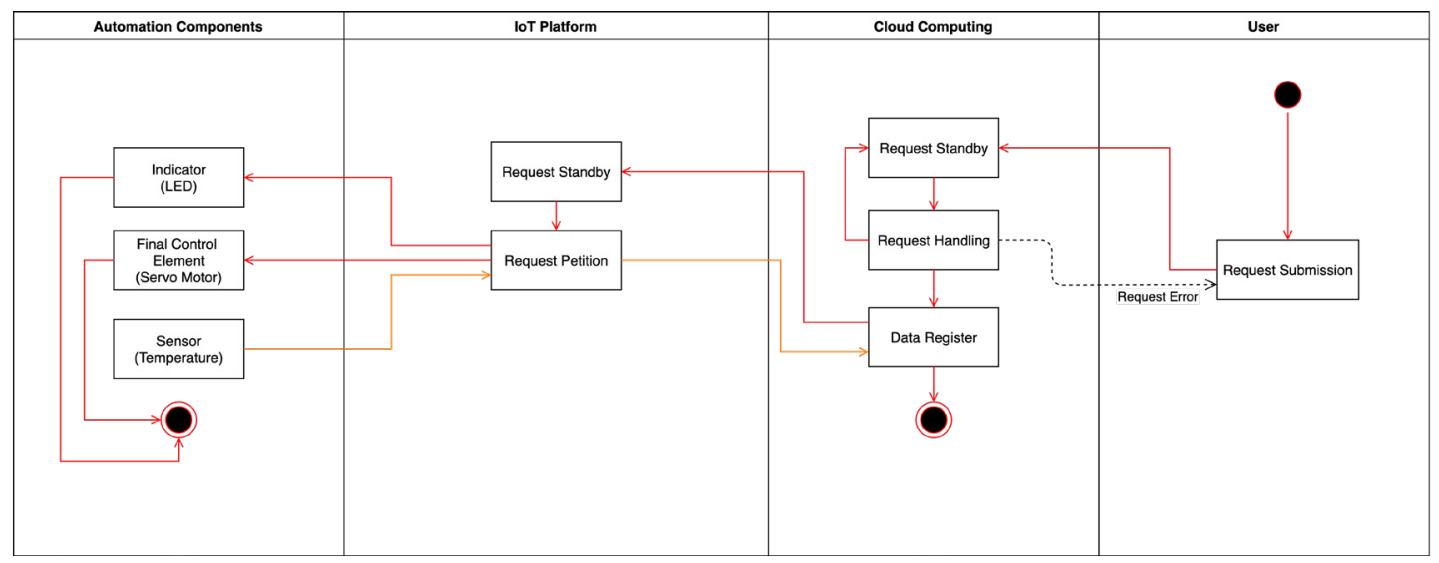

Figure 4. Diagram of Internet of Things request

The request is sent and recorded in the cloud, where it is executed and sent to the automation elements integrated into the platform, which execute them. In the case of a sensor, the platform acquires the data and sends them for recording in the cloud.

\section{Results and Analysis}

The results are displayed based on the different scenarios that were assessed.

\subsection{Scenario: totally disconnected}

The totally disconnected scenario features a reduction in consumption and sending of data to the cloud, because it is not mandatory. Having described the above, tests are performed to establish the indication, handling and storage of the data acquired by the Fog platform. Figure 3 displays a report of the data in the Fog platform, which generates a reduction in the use of the Internet service and a reduction in the use of the IoT platform.

\subsection{Scenario: Compare IoT and Fog Computing}

For the scenario of comparing both architectures, the IoT framework called Cayenne was selected (Artono $\&$ Susanto, 2017). The sensors and indicators are connected to this IoT tool, and measurements are made using a framework to capture requests by DNS. The received and sent requests are measured, in order to measure the requests made to the cloud. In this case, the cloud is the address of the IoT platform. This measurement was taken by placing a DNS service in a broker that connects, when required, to the IoT platform and to the Fog Platform. The devices are located within a local network and assigned to the external DNS service to collect the number of petitions inside and outside the local network, and records them for subsequent analysis.

Afterwards, a five-day time frame was established to perform measurements and collect the number of requests generated by the Raspberry Pi device to the IoT platform. This does not include the size of the packages nor the time it takes them to arrive at the destination, due to the project's approach.

Figure 5 displays the measurement trends, indicating the number of petitions by time and day. It indicates that behavior is not constant, but on demand, i.e., if the service is required it is recorded and sent to perform the task. Figure 6 displays the various domains and the number of requests during the defined time period. The results display a high volume of requests to the IoT platform, which confirms the high demand for access to the platform, due to the IoT approach. Due to the above, the relevant measurements of network traffic display an advantage for Fog Computing in terms of a $70.53 \%$ reduction in requests to IoT, which is confirmed based on the implementation of the architecture. It additionally confirms the findings of other authors who indicate a reduction in processing, web services and communications to the IoT platform. 


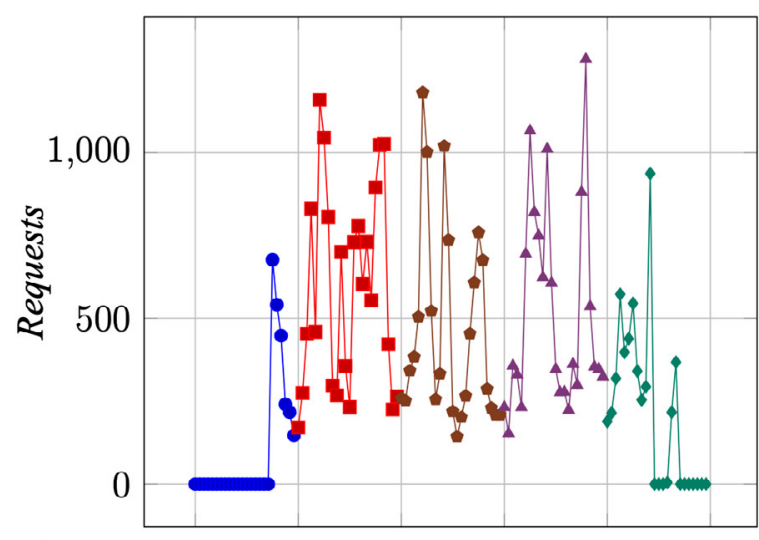

- Day $1-$ Day $2-$ Day $3 \backsim$ Day $4-$ Day 5

Figure 5. Kequests over 5 days

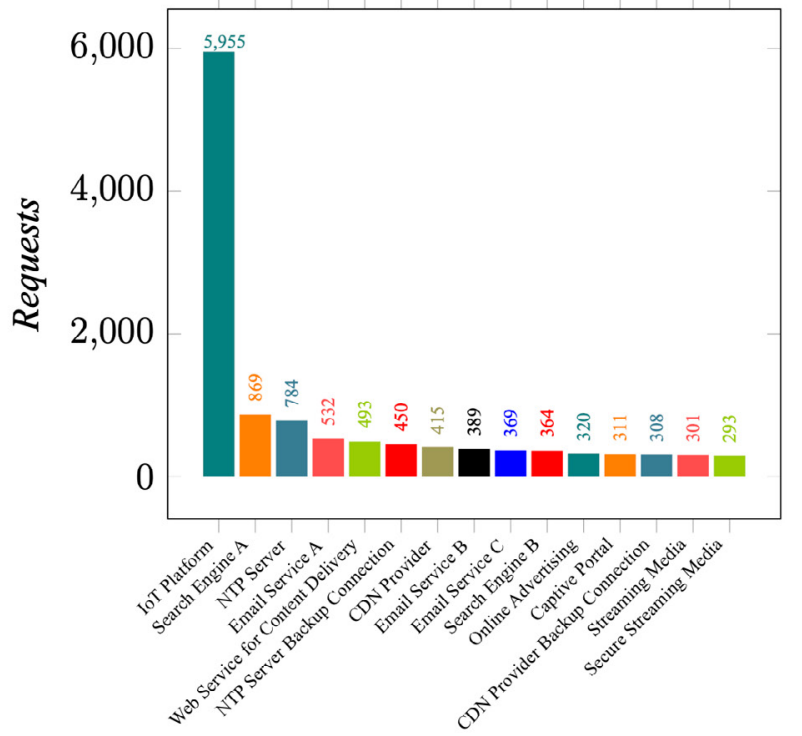

Figure 6. Service requests

The above results demonstrate that the Fog Computing platform is a complement for cloud computing and a new approach towards the Internet of Everything.

\section{Conclusions and Recommendations}

This paper establishes a frame of reference for developing and implementing a Fog Computing architecture for the effects of concept testing, with the hardware and software tools and with web management and abstract and intuitive resources that facilitate understanding, thereby obtaining new ideas and intellectual resources to produce alternatives for both hardware and software for the implementation of this new trend in the academic environment.

All the modules used to this effect fulfill the project's proposed objectives. The result obtained is consistent with the findings of the literature. In general, it was observed that sensor data was processed before being transported to the IoT platform, and that the data is independent.

It is important to select hardware that enables interaction with the various software modules. It is suggested to follow the research steps that integrate the various types of modules, such as communications, acquisition, processing and viewing of data, user management and expansion of the information security layer. 


\section{Acknowledgments}

The authors wish to thank the assistance of all partners of the project Centro de Excelencia y Apropiacion en Internet de las Cosas (CEA-IoT). The authors also wish to thank all the institutions that supported this study: the Colombian Ministry of Information and Communications Technology (MinTIC) and the Administrative Department of Science, Technology and Innovation (Colciencias), through the Francisco José de Caldas National Financing Fund for Science, Technology and Innovation (Project ID: FP44842502-2015).

\section{Statement of conflicts of interest}

The authors declared no potential conflicts of interest with respect to the research, authorship and/or publication of this article.

\section{References}

Artono, B., \& Susanto, F. (2017). LED control system with cayenne framework for the Internet of Things (IoT). JEECAE (Journal of Electrical, Electronics, Control, and Automotive Engineering), 2(1), 95-100.

Bakhshi, Z., Rodriguez-Navas, G., \& Hansson, H. (2019). Dependable Fog Computing: A Systematic Literature Review. 2019 45th Euromicro Conference on Software Engineering and Advanced Applications (SEAA), 395-403. https://doi.org/10.1109/SEAA.2019.00066

Homekit. (2016). Retrieved January 2, 2017, from https://www.domoticz.com/wiki/Homekit_Siri

Iorga, M., Feldman, L., Barton, R., Martin, M. J., Goren, N. S., \& Mahmoudi, C. (2018). Fog Computing Conceptual Model. Retrieved from https://www.nist.gov/publications/fog-computing-conceptual-model

IoT Eclipse. (2019). IoT Developer Survey. Retrieved from Eclipse Foundation website: https://iot.eclipse.org/ resources/iot-developer-survey/iot-developer-survey-2019.pdf

Isa, I. S. M., Musa, M. O. I., El-Gorashi, T. E. H., \& Elmirghani, J. M. H. (2019). Energy Efficient and Resilient Infrastructure for Fog Computing Health Monitoring Applications. 2019 21st International Conference on Transparent Optical Networks (ICTON), 1-5. https://doi.org/10.1109/ICTON.2019.8840438

Skala, K., Davidovic, D., Afgan, E., Sovic, I., \& Sojat, Z. (2015). Scalable distributed computing hierarchy: Cloud, fog and dew computing. Open Journal of Cloud Computing (OJCC), 2(1), 16-24.

Tsigkanos, C., Avasalcai, C., \& Dustdar, S. (2019). Architectural Considerations for Privacy on the Edge. IEEE Internet Computing, 23(4), 76-83. https://doi.org/10.1109/MIC.2019.2935800

Wang, X., Gu, B., Ren, Y., Ye, W., Yu, S., Xiang, Y., \& Gao, L. (2019). A Fog-based Recommender System. IEEE Internet of Things Journal, 1-1. https://doi.org/10.1109/JIOT.2019.2949029

Wei, X., \& Wu, L. (2019). A New Proposed Sensor Cloud Architecture Based on Fog Computing for Internet of Things. 2019 International Conference on Internet of Things (IThings) and IEEE Green Computing and Communications (GreenCom) and IEEE Cyber, Physical and Social Computing (CPSCom) and IEEE Smart Data (SmartData), 615-620. https://doi.org/10.1109/iThings/GreenCom/CPSCom/ SmartData.2019.00120

Zhang, T., Jin, J., Zheng, X., \& Yang, Y. (2019). Rate Adaptive Fog Service Platform for Heterogeneous IoT Applications. IEEE Internet of Things Journal, 1-1. https://doi.org/10.1109/JIOT.2019.2945328 